\title{
Propriedades Reométricas e Mecânicas e Morfologia de Compósitos Desenvolvidos com Resíduos Elastoméricos Vulcanizados
}

\author{
Aline Zanchet, Nicolle Dal'Acqua, Tatiana Weber, Janaina S. Crespo \\ Departamento de Física e Química, UCS \\ Rosmary N. Brandalise \\ Departamento de Engenharia Química, UCS \\ Regina C. R. Nunes \\ Instituto de Macromoléculas, UFRJ
}

\begin{abstract}
Resumo: Uma alternativa para as empresas geradoras de resíduos elastoméricos é a incorporação dos mesmos em suas formulações. Esse trabalho tem como objetivo preparar compósitos a partir da incorporação de resíduos industriais de SBR (copolímero de butadieno-estireno) e de EPDM (terpolímero de etileno-propileno-dieno), em formulação ASTM específica para cada tipo de elastômero. Foram avaliadas as propriedades reométricas, mecânicas e morfológicas dos compósitos desenvolvidos. A incorporação do resíduo permitiu a obtenção de produtos que vulcanizam em menores tempos quando comparados a composições sem resíduo. A quantidade de resíduo, para o melhor resultado de resistência à tração foi de 37 e de 196 phr para os compósitos com SBR e EPDM, respectivamente. As micrografias de MEV corroboraram os resultados mecânicos dessas composições, indicando melhor homogeneidade do resíduo na respectiva matriz elastomérica.
\end{abstract}

Palavras-chave: Resíduo de SBR, resíduo de EPDM, compósitos elastoméricos, propriedades mecânicas, reometria.

\section{Cure Characteristics, Mechanical Properties and Morphology of Composites Developed with Addition of Elastomeric Vulcanized Ground Scraps}

\begin{abstract}
One alternative for elastomeric scraps generation is its incorporation in conventional formulations in the industry itself. In this work, compositions with incorporation of SBR (styrene butadiene rubber) and EPDM (ethylene propylene diene rubber) industrial scraps, in specific ASTM formulation for each elastomer, were prepared. Rheometric characteristics, mechanical properties and the morphology of the compounds developed were evaluated. With the ground scraps incorporation the vulcanization time decreases, comparing with compounds without ground scraps. The amount of scraps for the best tensile strength properties was 37 and $196 \mathrm{phr}$ for SBR and EPDM compositions, respectively. Micrographs corroborated the mechanical results, indicating the best homogeneity of the scraps in the elastomeric matrix for these compositions.
\end{abstract}

Keywords: SBR ground scraps, EPDM ground scraps, elastomeric compounds, mechanical properties, cure characteristics.

\section{Introdução}

Artefatos elastoméricos são utilizados nas mais diferentes indústrias como a automobilística, a calçadista, a hospitalar, entre outras. A ampla utilização em pneumáticos tem como conseqüência, a grande geração de resíduos pós-consumo que constitui um sério problema ambiental ${ }^{[1,2]}$.

É de conhecimento que os polímeros termoplásticos apresentam degradação lenta e que nos elastômeros esta degradação lenta é potencializada pela presença de ligações cruzadas e de outros aditivos como antioxidantes, antiozonantes, que dificultam o seu reaproveitamento ${ }^{[1]}$. Segundo Sreeja ${ }^{[3]}$, apenas 8 a $12 \%$ dos resíduos poliméricos são de polímeros termoplásticos, enquanto que o restante são elastômeros, representados principalmente pelos pneus pós-consumo ${ }^{[4-7]}$.
Uma forma de reutilização desses resíduos elastoméricos, após transformá-los em pó, é como carga em novas formulações. Este procedimento traz como vantagem o baixo custo, mas, segundo a literatura, apenas pequenas quantidades podem ser adicionadas à borracha virgem sem causar perda de propriedades, devido à fraca interação borracha vulcanizada-borracha virgem ${ }^{[1,2,8,9]}$.

No caso de resíduos gerados pela própria indústria do artefato, cuja origem e formulação é conhecida, a incorporação dos mesmos em elastômeros virgens torna-se atraente no desenvolvimento de composições com propriedades de interesse tecnológico, que acarretam também em economia para a empresa.

Este trabalho tem como objetivo preparar compósitos a partir da incorporação de resíduos industriais de SBR e de 
EPDM, em formulação ASTM específica para cada tipo de elastômero, e avaliar as propriedades reométricas, mecânicas e morfológicas dos materiais obtidos.

\section{Experimental}

\section{Coleta dos resíduos}

Amostras de aparas de perfis expandidos de SBR e de EPDM, respectivamente, foram coletadas segundo a norma NBR $10007^{[10]}$, na empresa CIAFLEX Indústria de Borrachas Ltda, em Caxias do Sul, RS, entre os dias 30/08/2004 e 18/09/2004. Foram coletados $35 \mathrm{~kg}$ de SBR e $25 \mathrm{~kg}$ de EPDM.

\section{Moagem dos resíduos}

A moagem dos resíduos das amostras de SBR e EPDM foi realizada nos equipamentos disponíveis no Laboratório de Polímeros da Universidade de Caxias do Sul. Como as aparas eram extremamente heterogêneas em tamanho e forma, primeiramente as mesmas foram serradas em uma serra-fita, num comprimento de aproximadamente $10 \mathrm{~cm}$, na seqüência foram submetidas a um aglutinador. Depois desta primeira etapa, as amostras foram submetidas a um misturador de rolos $\mathrm{MH}$, modelo $\mathrm{MH}-600 \mathrm{C}$. Na etapa seguinte, a desfragmentação das amostras foi realizada em um misturador basculante $\mathrm{MH}$ modelo $\mathrm{MH}-4$. Por fim o resíduo foi triturado no moinho de bancada, obtendo-se assim o resíduo de SBR moído (SBR-r) e o resíduo de EPDM moído (EPDM-r) ${ }^{[11,12]}$.

A granulometria, determinada para o SBR-r e para o EPDM-r, ficou entre 0,21 a 0,41 mm que, segundo literatu$\mathrm{ra}^{[8]}$, está na faixa aceitável para a incorporação em composições poliméricas. Assim, o processo de moagem realizado no laboratório foi efetivo e produziu um pó adequado ao estudo proposto. O teor de borracha em cada resíduo foi determinado por análise térmica, sendo de 30,5\% para o SBR-r e de $21,8 \%$ para o EPDM-r ${ }^{[11,12]}$.

\section{Preparação dos compósitos elastoméricos}

Os compósitos elastoméricos foram preparados em um misturador de rolos de laboratório, modelo MH-600C, da MH Equipamentos, segundo norma ASTM D 3185-99 ${ }^{[13 a]}$ e ASTM D 3568-98 ${ }^{[13 \mathrm{~b}]}$, respectivamente, para o SBR e o EPDM. Para efeito comparativo, composições sem a adição de resíduo foram preparadas, sendo denominadas simplesmente por SBR e EPDM.

O SBR utilizado foi o tipo 1502 da PETROFLEX Indústria e Comércio S/A. Foram empregados dois tipos de EPDM o 4770 e o 4703 da DSM South América Ltda, com teores de etileno de $70,3 \%$ e $53,0 \%$, respectivamente. Os outros aditivos utilizados foram os seguintes: óxido de zinco (AGRO ZINCO Indústria e Comércio Ltda); enxofre (INTERCUF Indústria e Comércio Ltda); ácido esteárico, N-terc-butil-2benzotiazol sulfenamida (TBBS), dissulfeto de tetrametiltiuram (TMTD) e mercaptobenzotiazol (MBT) (PROQUIEC Indústria de Produtos Químicos S/A). Todos os materiais foram utilizados como recebidos, e as formulações desenvolvidas estão apresentadas na Tabela 1.

\section{Determinação dos parâmetros de vulcanização (reômetro de disco oscilatório)}

Os parâmetros de vulcanização foram obtidos em um reômetro de disco oscilatório Tech Pro, modelo Rheotech OD+, com freqüência de $100 \mathrm{cpm}$, amplitude de deformação de $1^{\circ}$ e temperatura de $160{ }^{\circ} \mathrm{C}$. Os testes foram realizados segundo ASTM D 2084-95 $5^{[13 c]}$.

\section{Obtenção dos corpos de prova e ensaios mecânicos}

Placas com dimensões de $160 \times 160 \times 1,5 \mathrm{~mm}$, foram vulcanizadas a $160{ }^{\circ} \mathrm{C}$ e pressão de $7,5 \mathrm{MPa}$, segundo norma ASTM D 3182-89 ${ }^{[13 \mathrm{~d}]}$, nos tempos de vulcanização $\left(\mathrm{t}_{90}\right)$ respectivos para cada formulação, conforme indicado pelo reômetro de disco oscilatório. A partir dessas placas, foram cortados corpos de prova específicos para os ensaios mecânicos propostos: resistência à tração (ASTM D 412-98 ${ }^{[13 e]}$ ), resistência ao rasgamento, (ASTM D 624-98 ${ }^{[13 \mathrm{f}]}$ ) e dureza Shore A (ASTM D 2240-97 $\left.{ }^{[13 \mathrm{~g}]}\right)$. Para a realização dos ensaios mecânicos os corpos de prova foram acondicionados em temperatura de 23 $\pm 2{ }^{\circ} \mathrm{C}$ e umidade relativa de $50 \pm 5 \%$ durante 48 horas.

\section{Microscopia eletrônica de varredura}

As micrografias dos compósitos preparados foram obtidas em um Microscópio Eletrônico de Varredura (MEV) PHILIPS XL 30, com voltagem de aceleração de $20 \mathrm{kV}$. As amostras foram fraturadas criogenicamente e previamente recobertas com $\mathrm{Au}$, antes de serem analisadas.

\section{Resultados e Discussão}

Os parâmetros reométricos para os compósitos desenvolvidos estão apresentados na Tabela 2.

$\mathrm{O}$ torque mínimo, $\mathrm{M}_{\mathrm{L}}$, está relacionado à viscosidade da composição na temperatura do ensaio, e, dessa forma, é um indicativo da processabilidade ${ }^{[14]}$. Observa-se que o $M_{L}$ aumenta com o teor de resíduo, tanto para os compósitos com SBR quanto para os com EPDM. No caso do compósito EPDM/

Tabela 1. Formulações dos compósitos SBR/SBR-r e EPDM/EPDM-r.

\begin{tabular}{lrrrrrr}
\hline Componentes (phr) & \multicolumn{7}{c}{ Formulações } \\
\hline SBR 1502 & 100 & 100 & 100 & 100 & - & - \\
SBR-r & 0 & 37 & 83 & 141 & - & - \\
EPDM 4770/4703 & $64 / 36$ & $64 / 36$ & $64 / 36$ & $64 / 36$ & $64 / 36$ & $64 / 36$ \\
EPDM-r & 0 & 51 & 115 & 196 & 305 & 458 \\
\hline
\end{tabular}

${ }^{\text {a} F o r m u l a c ̧ a ̃ o ~ A S T M ~ D ~ 3185-99 ~}{ }^{[13 a]}$, em phr: ZnO 3; ácido esteárico 1; TBBS (N-terc-butil-2 benzotiazol sulfenamida) 1; enxofre 1,75; ${ }^{\text {FFormulação }}$ ASTM D 3568-98 ${ }^{[13 b]}$, em phr: ZnO 5; ácido esteárico 1; TMTD (dissulfeto de tetrametiltiuram) 1; MBT (mercaptobenzotiazol) 0,5; enxofre 1,50. 
Tabela 2. Parâmetros reométricos dos compósitos SBR/SBR-r e EPDM/EPDM-r.

\begin{tabular}{lcccc}
\hline Compósito & $\mathbf{M}_{\mathbf{L}}(\mathbf{d N} \mathbf{~ m})^{\mathbf{a}}$ & $\mathbf{M}_{\mathbf{H}}(\mathbf{d N} \mathbf{~ m})^{\mathbf{b}}$ & $\Delta \mathbf{M}(\mathbf{d N} \mathbf{~ m})^{\mathbf{c}}$ & $\mathbf{t}_{\mathbf{s 1}}(\mathbf{m i n})^{\mathbf{d}}$ \\
\hline SBR/SBR-r & & & & 6,08 \\
$100 / 0$ & 9,34 & 65,62 & 56,28 & 4,14 \\
$100 / 37$ & 11,10 & 55,71 & 44,61 & 2,40 \\
$100 / 83$ & 15,30 & 31,62 & 16,32 & 2,23 \\
$100 / 141$ & 19,28 & 33,46 & 14,18 & 3,42 \\
EPDM/EPDM-r & & & & 2,00 \\
$100 / 0$ & 7,83 & 75,53 & 67,70 & 1,35 \\
$100 / 51$ & 11,96 & 74,60 & 62,64 & 1,19 \\
$100 / 115$ & 17,55 & 71,73 & 54,18 & 1,05 \\
$100 / 196$ & 19,38 & 60,54 & 41,16 & 1,18 \\
$100 / 305$ & 19,78 & 47,78 & 28,00 & 35,72 \\
$100 / 458$ & 15,03 & 50,75 & & \\
\hline
\end{tabular}

${ }^{\mathrm{a}} \mathrm{M}_{\mathrm{L}}$ : Torque mínimo; ${ }^{\mathrm{b}} \mathrm{M}_{\mathrm{H}}$ : torque máximo; ${ }^{\mathrm{c}} \Delta \mathrm{M}$ : diferença entre os torques $\left(\mathrm{M}_{\mathrm{H}}-\mathrm{M}_{\mathrm{L}}\right)$; e ${ }^{\mathrm{d}} \mathrm{t}_{\mathrm{s} 1}$ : tempo de segurança de processo.

EPDM-r 100/458, há um decréscimo, e esse comportamento é indicativo do excesso de resíduo nessa formulação.

O torque máximo, $\mathrm{M}_{\mathrm{H}}$, está relacionado à formação de ligações cruzadas. Os resultados da Tabela 2 para esse parâmetro indicam um decréscimo de $\mathrm{M}_{\mathrm{H}}$ com o aumento de resíduo, relacionado à diminuição do teor da borracha virgem onde as ligações cruzadas estão se formando ${ }^{[9,14]}$. Um outro fator interessante a ser analisado é a diferença entre os torques, $\Delta \mathrm{M}\left(\mathrm{M}_{\mathrm{H}}-\mathrm{M}_{\mathrm{L}}\right)$, como um indicativo da influência do resíduo no compósito. Observa-se que esse valor diminui com a adição do resíduo para ambos os elastômeros estudados, sendo o decréscimo mais acentuado para a formulação SBR/SBR-r 100/141 e EPDM/EPDM-r 100/305, indicando valores limites de resíduo para as respectivas formulações.

$\mathrm{O} \mathrm{t}_{\mathrm{s} 1}$ é um parâmetro importante fornecido pelo reômetro de disco oscilatório, pois indica o tempo de segurança de processo, isto é, o tempo limite para o início da formação de ligações cruzadas. Os resultados de todos os compósitos preparados indicam um decréscimo nesse valor com o aumento da incorporação de resíduo, quando os valores são comparados às composições de SBR ou EPDM sem resíduo. Esses resultados foram observados anteriormente ${ }^{[9,14]}$, e são esperados uma vez que nos resíduos há a presença do sistema de aceleração residual. Esses resultados permitem que os artefatos sejam obtidos em menor tempo, quando a comparação é feita com as formulações sem resíduo, acarretando dessa forma em uma economia no processo.

Os resultados mecânicos realizados com os compósitos desenvolvidos estão descritos na Tabela 3.

Observa-se que a dureza apresentou o mesmo comportamento do torque máximo, isto é, diminui com o aumento da incorporação de resíduo. Esses resultados podem ser comparados uma vez que tanto a dureza quanto o torque máximo estão associados à rigidez do material. Um outro parâmetro relacionado ao aumento de rigidez molecular proveniente da adição dos resíduos vulcanizados é o módulo a baixas deformações (módulo a 100\% de alongamento), que apresenta o mesmo comportamento observado para dureza e torque máximo ${ }^{[15]}$.

A resistência à tração e ao rasgamento são propriedades importantes na determinação do melhor desempenho mecânico de formulações elastoméricas. Os resultados de resistência ao rasgamento dos compósitos de SBR desenvolvidos são menores do que o da formulação sem carga. Analisando somente os compósitos com resíduo, o que mostrou o me-

Tabela 3. Propriedades mecânicas dos compósitos SBR/SBR-r e EPDM/EPDM-r.

\begin{tabular}{ccccc}
\hline Compósito & Dureza (Shore A) & $\begin{array}{c}\text { Módulo a 100\% } \\
(\mathbf{M P a})\end{array}$ & $\begin{array}{c}\text { Energia de ruptura } \\
(\mathbf{J})\end{array}$ & $\begin{array}{c}\text { Resistência ao rasgamento } \\
\left(\mathbf{k N . m} \mathbf{m}^{-1}\right)\end{array}$ \\
\hline $\begin{array}{c}\text { SBR/SBR-r } \\
\text { 100/0 }\end{array}$ & $45 \pm 3$ & $1,07 \pm 0,09$ & $0,88 \pm 0,18$ & $16,4 \pm 0,91$ \\
$100 / 37$ & $47 \pm 2$ & $0,93 \pm 0,08$ & $1,23 \pm 0,10$ & $13,1 \pm 1,94$ \\
$100 / 83$ & $44 \pm 2$ & $0,60 \pm 0,05$ & $2,09 \pm 0,19$ & $13,5 \pm 1,29$ \\
$100 / 141$ & $43 \pm 2$ & $0,58 \pm 0,04$ & $1,51 \pm 0,11$ & $11,9 \pm 1,78$ \\
\hline EPDM/EPDM-r & & & & $8,90 \pm 1,10$ \\
$100 / 0$ & $47 \pm 1$ & $0,94 \pm 0,08$ & $0,69 \pm 0,23$ & $12,9 \pm 0,83$ \\
$100 / 51$ & $46 \pm 1$ & $1,06 \pm 0,04$ & $1,04 \pm 0,11$ & $14,5 \pm 1,66$ \\
$100 / 115$ & $44 \pm 1$ & $0,97 \pm 0,05$ & $1,65 \pm 0,11$ & $13,8 \pm 1,24$ \\
$100 / 196$ & $42 \pm 1$ & $0,87 \pm 0,07$ & $2,11 \pm 0,33$ & $13,8 \pm 1,44$ \\
$100 / 305$ & $37 \pm 2$ & $0,73 \pm 0,03$ & $1,67 \pm 0,18$ & $13,7 \pm 2,03$ \\
$100 / 458$ & $35 \pm 1$ & $0,69 \pm 0,03$ & $1,53 \pm 0,33$ & \\
\hline
\end{tabular}


lhor resultado foi o da formulação SBR/SBR-r 100/83. Esse resultado pode ser corroborado pelo valor de energia de ruptura, que é a área sob a curva de tensão versus deformação, encontrado para essa formulação. No caso dos compósitos EPDM/EPDM-r, os resultados de resistência ao rasgamento são maiores do que o da formulação sem resíduo, sendo as formulações 100/115 e 100/196 as que apresentaram os melhores desempenhos. Esses resultados corroboram os valores encontrados para energia de ruptura indicando como o compósito mais resistente, o EPDM/EPDM-r 100/196.

Nas Figuras 1a e 1b estão apresentados os valores de tração e alongamento na ruptura para as formulações com SBR e EPDM, respectivamente.

A quantidade ótima de resíduo nos compósitos desenvolvidos pode ser justificada pelos valores máximos de tração na ruptura. A Figura 1a indica a formulação de SBR/SBR-r 100/37 e a Figura 1b, a formulação EPDM/EPDM-r 100/196.

Conforme discutido anteriormente, quando a comparação é feita com as composições sem carga, o aumento do teor de resíduo leva a valores menores de rigidez molecular, refletindo no aumento do alongamento na ruptura para todos os compósitos preparados.

Na Figura 2 estão apresentadas as micrografias de MEV das superfícies fraturadas dos compósitos desenvolvidos com

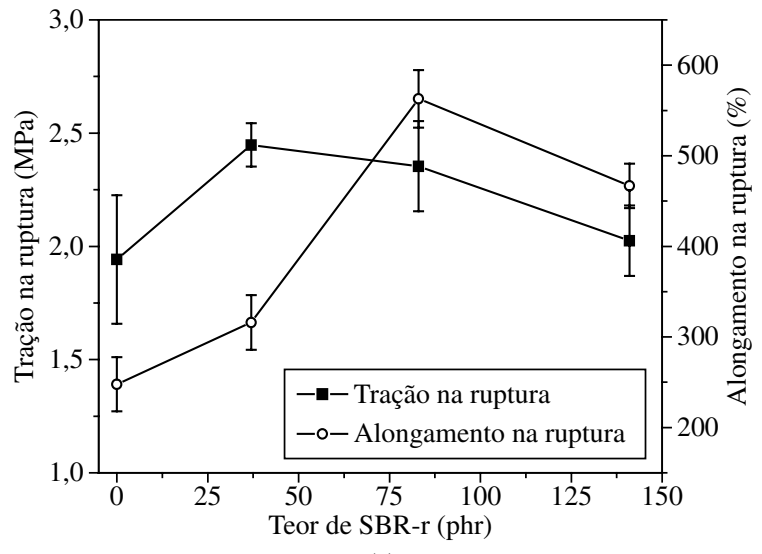

(a)

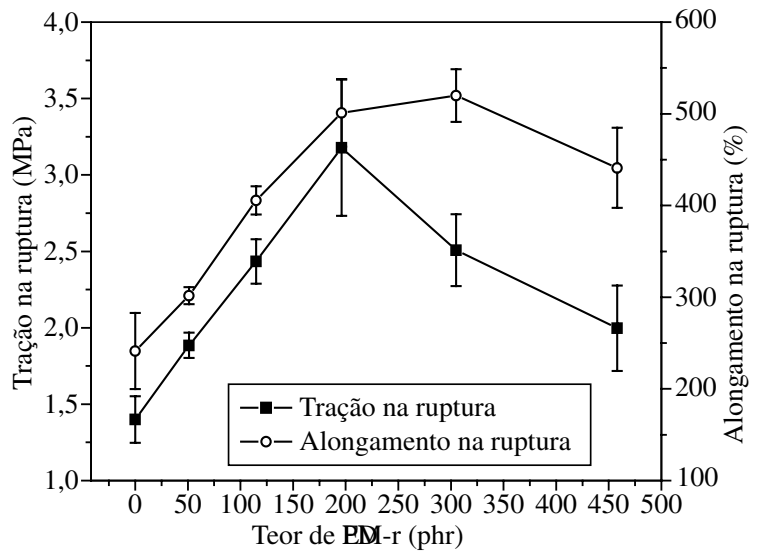

(b)

Figura 1. Resistência à tração e alongamento na ruptura dos compósitos. a) SBR/SBR-r; e b) EPDM/EPDM-r.
SBR-r (Figuras 2a e 2b) e com EPDM-r (Figuras 2c e 2d). Os compósitos desenvolvidos mostram pelo menos duas fases, apresentando heterogeneidade na distribuição das mesmas, sendo o resíduo o constituinte da fase mais clara ${ }^{[15]}$.

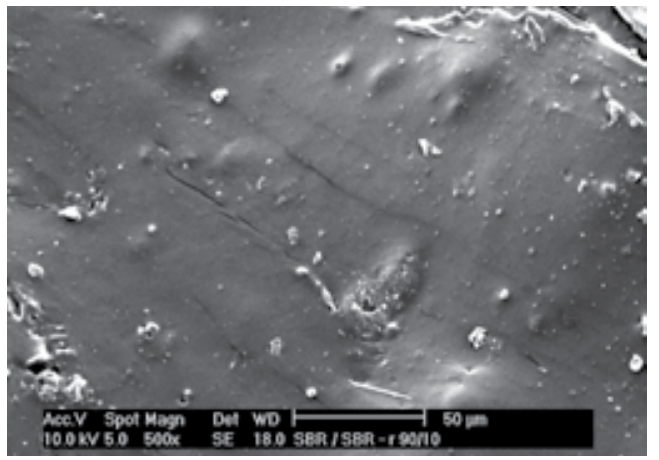

(a)

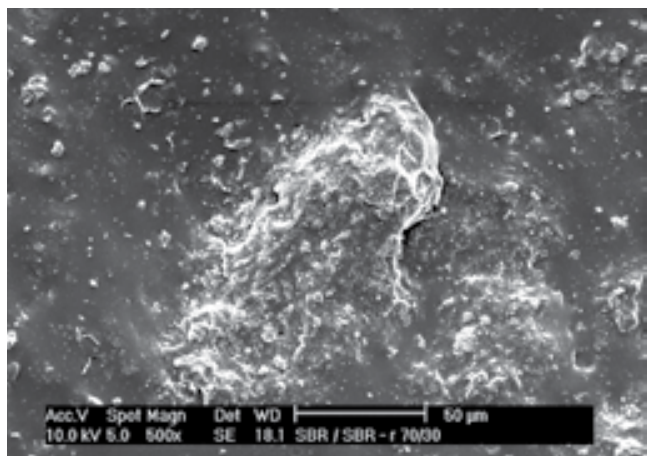

(b)

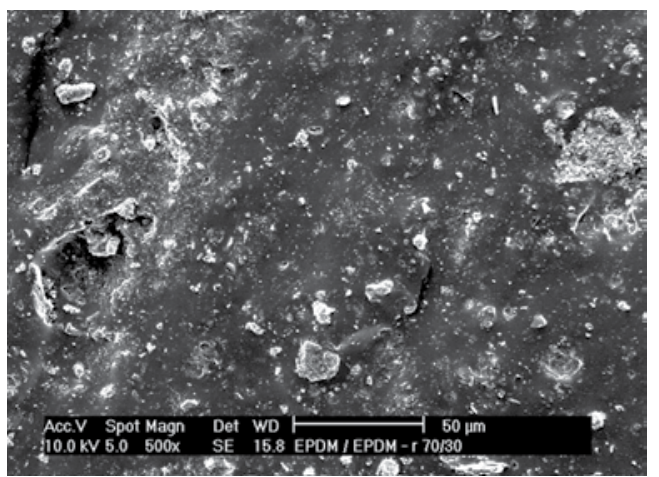

(c)

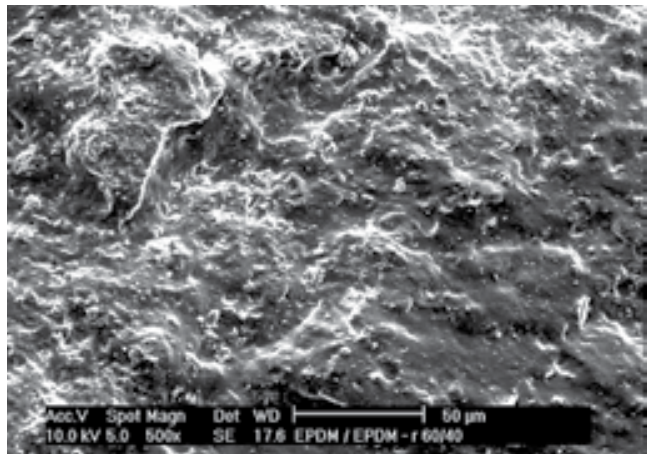

(d)

Figura 2. Fotomicrografias das composições desenvolvidas em phr. a) SBR/ SBR-r 100/37; b) SBR/SBR-r 100/141; c) EPDM/EPDM-r 100/196; e d) EPDM/EPDM-r 100/305. Aumento 500 vezes. 
As micrografias dos compósitos com os melhores resultados de resistência à tração na ruptura, SBR/SBR-r 100/37 (Figura 2a) e EPDM/EPDM-r 100/196 (Figura 2c), apresentaram uma maior homogeneidade e melhor distribuição matriz-resíduo. O aumento da quantidade de resíduo, nos compósitos desenvolvidos, provocou o aparecimento de domínios e o descolamento do resíduo da matriz de SBR (Figura 2b) e da matriz de EPDM, corroborando a perda na propriedade mecânica de resistência à tração na ruptura, após a adição de 83 e 305 phr de SBR-r e de EPDM-r, respectivamente.

\section{Conclusões}

Por meio da metodologia de moagem empregada neste trabalho foi possível obter um pó para realizar a incorporação de uma quantidade significativa de resíduos de EPDM e de SBR em composições com elastômeros virgens.

A presença do resíduo promoveu uma aceleração na formação de ligações cruzadas o que é interessante do ponto de vista de produção.

Os melhores resultados de resistência à tração na ruptura, em relação à quantidade limite de resíduo incorporado, foram nas formulações SBR/SBR-r 100/37 e EPDM/EPDMr 100/196. Esses compósitos se caracterizam por apresentar homogeneidade na distribuição do resíduo na matriz elastomérica.

Os resultados dos compósitos desenvolvidos permitem sugerir a reutilização dos resíduos, o que acarretará em economia na preparação do artefato final.

\section{Agradecimentos}

Os autores agradecem a FAPERGS/Procoredes II (Processo $\mathrm{n}^{\mathrm{o}}$ : 0518831) e ao $\mathrm{CNPq}$ pelo auxílio financeiro; a CIAFLEX Indústria de Borrachas Ltda. pelos materiais fornecidos.

\section{Referências Bibliográficas}

1. Adhikari, B.; De, D. \& Maiti, S. - Prog. Polym. Sci., 25, p. 909 (2000).

2. Myhre, M. \& MacKillop, D. A. - Rubber Chem. Technol., 75, p. 429 (2002).

3. Sreeja, T. D. - Polym. Plast. Tech. and Eng., 42, p. 236 (2003).

4. Swor, R. A.; Jensen, L. W. \& Budzol, M. - Rubber Chem. Technol., 53, p. 1215 (1980).

5. Accetta, A. \& Vergnaud, J. M. - Rubber Chem. Technol., 54, p. 302 (1981).
6. Accetta, A. \& Vergnaud, J. M. - Rubber Chem. Technol., 55, p. 328 (1982).

7. Accetta, A. \& Vergnaud, J. M. - Rubber Chem. Technol., 55, p. 961 (1982).

8. Ishiaku, U. S.; Chong, C. S. \& Ismail, H. - Polym. Testing, 18, p. 621 (1999).

9. Gibala, D. \& Hamed, G. R. - Rubber Chem. Technol., 67, p. 636 (1994).

10. Associação Brasileira de Normas Técnicas - "Amostragem de Resíduos Sólidos NBR 10007”, (2004).

11. Zanchet, A.; Weber, T.; Brandalise, R. N.; Nunes, R. C. R. \& Crespo, J. S. - "SBR reciclado: Caracterização e Reometria de Compósitos Elastoméricos", in: Anais do $8^{\circ}$ Congresso Brasileiro de Polímeros, p. 1093, Águas de Lindóia - SP, nov (2005).

12. Dal'Acqua, N.; Brandalise, R. N.; Nunes, R. C. R. \& Crespo, J. S. - "Caracterização de Resíduos de EPDM e sua Influência na Reometria de Compósitos", in: Anais do $8^{\circ}$ Congresso Brasileiro de Polímeros, p. 1137, Águas de Lindóia - SP, nov (2005).

13. American Society for Testing and Materials - (a) "Standard Test Methods for Rubber - Evaluation of SBR (Styrene-Butadiene Rubber) Including Mixtures with Oil ASTM D 3185-99"; (b) "Standard Test Methods for Rubber - Evaluation of EPDM (Ethylene-Propylene- Diene Terpolymers) Including Mixtures with Oil ASTM D 3568-98"; (c) "Standard Test Methods for Rubber Property - Vulcanization Using Oscillating Disk Cure Meter ASTM D 2084-95"; (d) "Practice for Rubber - Materials, Equipment, and Procedures for Mixing Standard Compounds and Preparing Standard Vulcanized Sheets ASTM D 3182-89"; (e) "Standard Test Methods for Vulcanized Rubber and Termoplastics Elastomers - Tension ASTM D 412-98a"; (f) "Standard Test Methods for Tear Strenght of Conventional Vulcanized Rubber and Termoplastics Elastomers ASTM D 624-98"; (g) "Standard Test Methods for Rubber Property - Durometer Hardness ASTM D 2240-97”, West Conshohocken (2000).

14. Nelson, P. A. \& Kutty, S. K. N. - Prog. in Rubber, Plast. and Recycl. Technol., 18, p. 85 (2002).

15. Farahani, T. D.; Bakhshandeh, G. R. \& Abtahi, M. Polym. Bulletin, 56, p. 495 (2006).

Enviado: 12/06/06

Reenviado: 04/10/06

Aceito: $11 / 10 / 06$ 